\title{
CASO 6-2014: Secuencia de Perfusión Arterial Reversa.
}

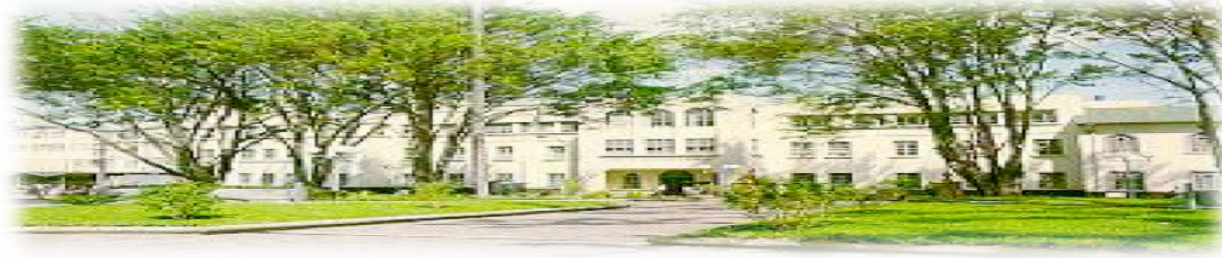

Hospital San quan de Dias. San Dosé. Costa Rica. Fundado en 1845

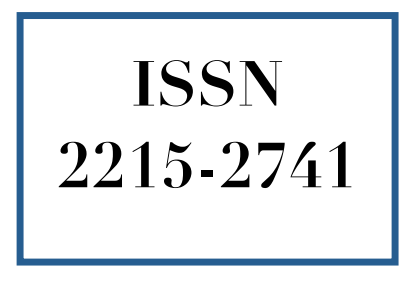

\section{Reporte de Caso}

Recibido: $\quad 12 / 11 / 2013$

Aceptado: $\quad$ 24/04/2014

\author{
Alejandro Cob Guillén ${ }^{1}$ \\ Joaquín Bustillos Villavicencio ${ }^{2}$ \\ Eugenio Calderón Solano ${ }^{2}$ \\ Róger Acevedo Riggioni ${ }^{2}$ \\ Jorge Mora Sandi ${ }^{2}$
}

${ }^{1}$ Médico General. Servicio de Ginecología y Obstetricia. Hospital San Juan de Dios. Correo electrónico: alecob00@hotmail.com

${ }^{2}$ Médicos Especialistas en Perinatología. Unidad de Perinatología. Hospital San Juan de Dios,. Correo electrónico: joaquin.bustillos@gmail.com

\section{RESUMEN}

La secuencia de perfusión arterial reversa (TRAP) es una malformación congénita exclusiva de gemelos monocoriales, con una incidencia aproximada de 1/35.000 embarazos. La malformación primaria es la ausencia de una estructura cardiaca en un gemelo, que se comporta como un parásito hemodinámicamente dependiente de su co-gemelo, a través de una anastomosis placentaria arterio-arterial. $\mathrm{La}$ historia natural conlleva una alta tasa de mortalidad perinatal del gemelo sano, principalmente por falla cardiaca y polihidramnios. Por su alta morbi-mortalidad, se ha intentado realizar intervenciones intraútero para minimizar complicaciones del gemelo normal, deteniendo la circulación del gemelo acárdico cuando está indicado. Se presenta un caso de una femenina de 30 años, obesa, tabaquista, hipertensa en control con enalapril y amlodipina, primigesta, con embarazo múltiple monocorial-biamniótico, en quien en ultrasonido control a los 4 meses de edad gestacional evidenció una malformación severa por un feto acárdico. La paciente requirió intervención intraútero a las 28 semanas de gestación, se realizó oclusión del cordón umbilical para feticidio selectivo del gemelo acárdico, guiado por ultrasonido.

\section{PALABRAS CLAVE}

TRAP. Embarazo Monocorial. Oclusión de Cordón 


\section{ABSTRACT}

The twin reversed arterial perfusion sequence (TRAP) is a rare complication unique to monozygotic multiple gestations. Occurs in approximately $1 / 35.000$ pregnancies. The malformation is characterized by the lack of a welldefined cardiac structure in one twin, which acts as a parasite, which is hemodynamically dependent on the normal twin, by a placental arterialarterial anastomosis. Without intervention, mortality rates for normal twins have been noted to be high, caused by heart failure or polyhydramnios. Because of the high degree of morbidmortality, it have been attempted to minimize complications to the normal twin by the occlusion of the acardiac twin umbilical cord when indicated. A 30 years old, obese, smoker, hypertense in treatment with enalapril and amlodipine, primigravida female, with a monochorionicbiamniotic multiple pregnancy, with an acardiac fetus sever malformation at 4 months of gestational age, diagnosed by an sonographic control test. She required intra uterus intervention at 28 week of gestational age, via cord occlusion selective feticide guided by sonography.

\section{KEY WORDS}

TRAP. Monochorionic Pregnancy. Cord Occlusion.

\section{CASO CLÍNICO}

Femenina de 30 años, obesa de $124 \mathrm{~kg}$, conocida hipertensa hace 7 años, en control en EBAIS, en tratamiento con enalapril y amlodipina, fumadora 28 paquetes/año. Con antecedente de infertilidad primaria en control en la clínica de esterilidad de Heredia, con embarazo múltiple sin terapia de inducción de la ovulación. Con una fecha de última menstruación del 2 de marzo, inició control prenatal en mayo, suspendió el fumado y cambió el tratamiento antihipertensivo pro alfa-metildopa. A los cuatro meses aproximadamente, se realizó un ultrasonido de control en clínica privada, donde se determinó una edad gestacional por US congruente con la fecha última menstruación. Pero se observó una malformación severa por un feto acárdico, es referida al Hospital San Juan de Dios (HSJD).
Al mes siguiente se realizó otro ultrasonido (24/8/11) donde se calculó el peso del gemelo B (acárdico) en 2984 g y el peso del gemelo A en $542 \mathrm{~g}$ por lo que se decidió interrumpir el embarazo del gemelo acárdico. Fue ingresada al HSJD el 5/9/11, con una edad gestacional de $26^{+4}$ semanas, para realizar el tratamiento. Se le realizó oclusión del cordón umbilical para feticidio selectivo del gemelo acárdico, guiada por ultrasonido. No se reportó complicaciones intraoperatorias, ni en el postoperatorio inmediato. Fue egresada el 22/9/11.

A las 31 semanas inició con salida de líquido transvaginal $(5 / 10 / 11), 4$ semanas posterior a tratamiento quirúrgico. Se le diagnosticó ruptura prematura de membranas, el ultrasonido no mostró datos de hidrops fetal, perfil biofísico $8 / 8$. Se le indicó su ingreso pero la paciente se rehúsa. El 6/10/11 acudió al servicio de emergencias por dolor abdominal intenso $\mathrm{y}$ salida de líquido transvaginal y fue ingresada con $31^{+1}$ semanas. Se le dió tratamiento con sulfato de magnesio, gentamicina y clindamicina. El 7/10/11 se le realizó cesárea por ruptura de membranas y corioamnioitis. El producto de la gesta fue una bebé con un peso de $1230 \mathrm{~g}$ y el gemelo acárdico de $1730 \mathrm{~g}$ al nacer.

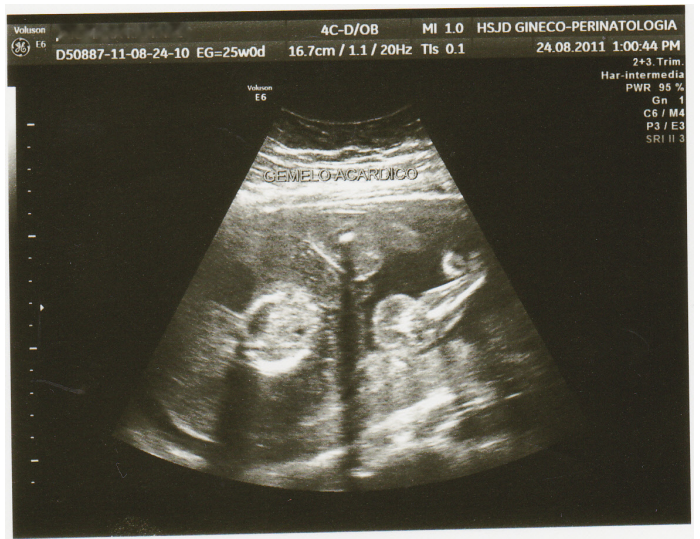

Imagen 1. Imagen ultrasonográfica de Secuencia TRAP, Unidad Perinatología, HSJD.

\section{DISCUSIÓN}

El embarazo múltiple se define como la condición en la que 2 o más fetos se desarrollan en la cavidad uterina durante una misma gestación. Tiene una incidencia de 1/80 
embarazos. Este se considera como una condición patológica, principalmente por la sobrecarga que produce. Se asocia a una mayor tasa de morbilidad y mortalidad perinatal. Los embarazos gemelares se pueden dividir en dos grandes grupos: los dicigotos y los monocigotos.

Los monocigotos tienen una incidencia de $25 \%$ y se pueden subdividir según el momento de la división $^{(1)}$ :
- Bicoriales-Biamnióticos: antes de $72 \mathrm{~h}$ : $30 \%$

- Monocoriales-Biamnióticos: $3^{\circ}$ día al $8^{\circ}$ día : $65 \%$

- Monocoriales-monoamnióticos: después del $8^{\circ}$ día: $5 \%$

Al tener una sola placenta, están asociados a patología por anastomosis de vasos, como el síndrome de transfusión feto-feto y la secuencia TRAP.

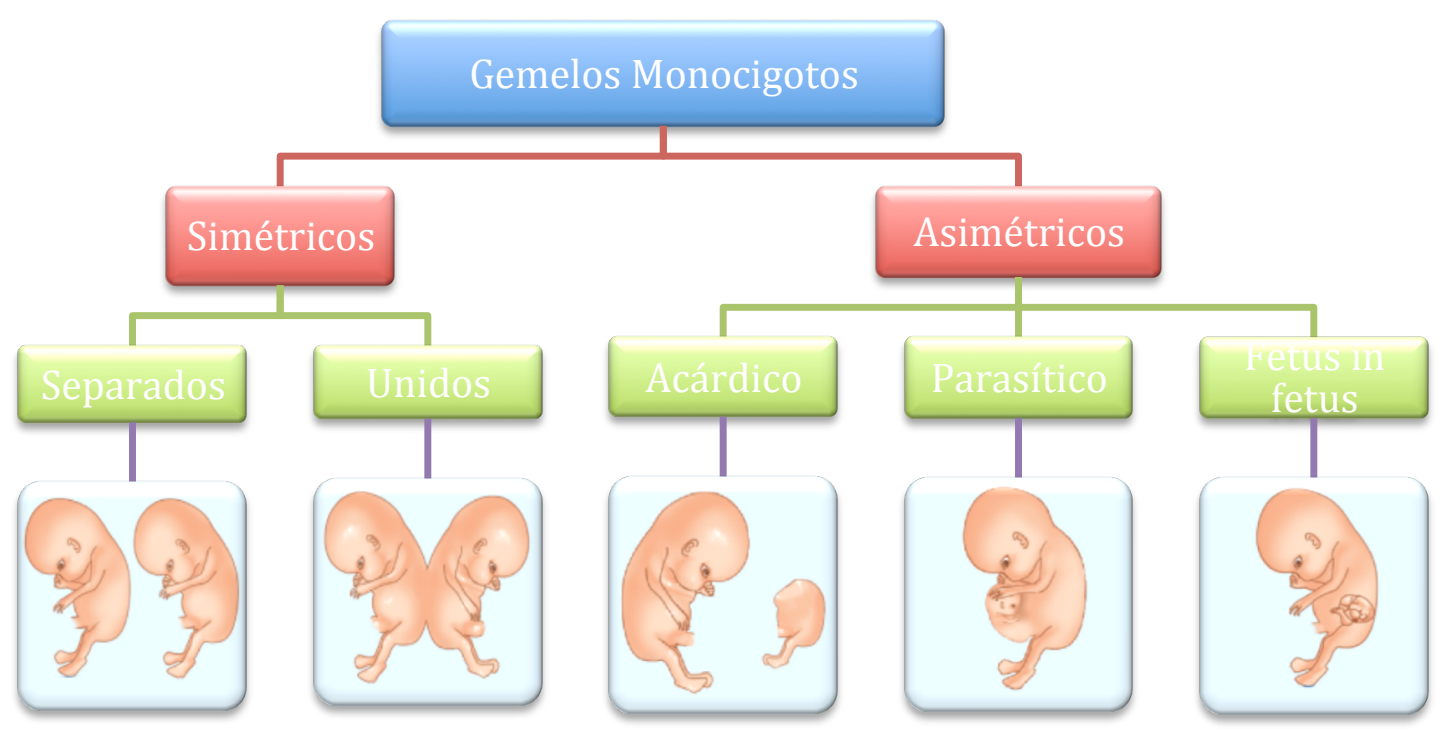

Imagen 2. Tipos de Gestación monocorial

La secuencia TRAP es una complicación exclusiva de las gestaciones múltiples monocoriales, que resulta del desarrollo de una malformación severa. La malformación primaria es la ausencia de una estructura cardiaca bien definida en un gemelo (gemelo acárdico), el cual es mantenido con vida por su co-gemelo (gemelo bomba) a través de una anastomosis placentaria arterio-arterial. El flujo de sangre arterial irriga de forma retrógrada las estructuras del acárdico, el cual se comporta como un parásito hemodinámicamente dependiente y continúa su crecimiento a expensas de la sangre aportada por su co-gemelo ${ }^{(2-5)}$.

Esta anomalía ocurre con una frecuencia de $1: 35.000$ embarazos y en un $0.3-1 \%$ de los embarazos gemelares monocoriales ${ }^{(1,3,5)}$, aunque en nuestro medio parece ser menos frecuente. $2 / 3$ ocurren en embarazos biamnióticos y $1 / 3$ en monoamnióticos. La historia natural de la enfermedad conlleva a la muerte del gemelo bomba en un $35-55 \%$ de los $\operatorname{casos}^{(2,5)}$.

\section{Etiología}

En el gemelo acárdico se encuentra afectado el desarrollo de todos los órganos y tiene una morfología muy variable, desde algunas estructuras bien diferenciadas hasta carecer de cualquier anatomía reconocible. En tamaño puede variar desde masas pequeñas que asemejan un teratoma hasta fetos de más del doble de su co-gemelo. El corazón puede ser completamente ausente (holoacardia) o estar en un estado primitivo de desarrollo (pseudoacardia). 
Frecuentemente el gemelo acárdico es acéfalo, con ausencia de extremidades superiores y en casi todos los casos se encuentra un tronco central con una columna bien desarrollada $o$ rudimentaria. En más de $2 / 3$ de los casos, presentan un cordón umbilical de 2 vasos. Los análisis citogenéticos no han demostrado ninguna alteración cromosómica consistente. Debido a la ausencia de comunicación entre los sistemas circulatorio y linfático, el gemelo acárdico frecuentemente desarrolla edema subcutáneo severo el cual puede alterar significativamente el tamaño del feto ${ }^{(2)}$.

En estos embarazos la placenta presenta una anastomosis arterio-arterial y otra veno-venosa que comunican las circulaciones de los gemelos. En muchos casos estas anastomosis se ubican en la superficie placentaria y conectan el cordón del gemelo acárdico con ramas arteriales y venosas del cordón del gemelo bomba. Ocasionalmente el cordón del acárdico se inserta en el cordón del su otro gemelo. Como consecuencia de esta conexión vascular, el gemelo acárdico siempre carece de tejido placentario funcional ${ }^{(2)}$.

\section{Fisiopatología}

La etiología y fisiopatología no han sido completamente aclaradas. Las dos principales teorías que intentan explicar el origen de la anomalía son $^{(2,5)}$ :

1. Vasculatura placentaria anormal que lleva a circulación reversa con alteración subsecuente del desarrollo cardiaco.

2. Embriogénesis cardiaca anormal como evento primario.

Según la secuencia TRAP, el flujo arterial sigue un curso en forma retrógrada por la arteria umbilical del gemelo acárdico a través de una anastomosis arterio-arterial única. Consecuentemente, la sangre escasa en nutrientes y pobremente oxigenada, saltándose la placenta, ingresa a la circulación del gemelo acárdico y pasivamente sigue un curso a través de las arterias iliacas a bajos niveles de presión para perfundir preferentemente las estructuras caudales más que las cefálicas. Este circuito explica el por qué las extremidades inferiores se encuentran con mejor desarrollo que las estructuras cefálicas, al recibir éstas sangre severamente desoxigenada, lo que produce el desarrollo anormal y atrofia del corazón y demás estructuras $^{(4)}$.

Teorías etiológicas alternativas han postulado que el evento primario es una alteración en la embriogénesis cardiaca que secundariamente causa flujo retrógrado. De acuerdo a la teoría que propone el desarrollo cardíaco, la supervivencia es debida únicamente a las anastomosis que se originan secundariamente ${ }^{(6)}$. Sin embargo, la demostración de latidos fetales en embarazos iniciales con posterior flujo umbilical reverso y desaparición de la actividad cardiaca, sugiere como defecto primario la anastomosis ${ }^{(7)}$.

Sin embargo la anastomosis arterio-arterial puede no estar presente en todos los gemelos acárdicos. Se ha visto casos de anastomosis arterio-venosas entre el gemelo acárdico y el gemelo bomba. En estos casos el flujo sanguíneo fue en sentido cefálico más que hacia miembros inferiores, pero las extremidades superiores permanecieron más deformes que las inferiores. La discordancia entre la teoría y lo observado en estos casos sugiere que el flujo retrógrado de sangre pobremente oxigenada puede no ser la única causa que explique el desarrollo ${ }^{(2)}$.

\section{Evolución}

Independientemente de la teoría, se sabe que el gemelo acárdico amenaza el bienestar de su cogemelo por un fenómeno de secuestro vascular. Hay tres mecanismos principales por los cuales aumenta la morbimortalidad del gemelo bomba ${ }^{(2)}$.

1. El crecimiento continúo del gemelo, que puede aumentar significativamente el volumen intrauterino y llevar a complicaciones perinatales derivadas como parto prematuro.

2. El shunt sistémico producido por el gemelo acárdico aumenta la demanda hemodinámica del gemelo bomba, pudiendo ocasionar insuficiencia cardiaca congestiva y polihidramnios.

3. La sangre desoxigenada del gemelo bomba queda más desoxigenada al perfundir la masa acárdica. Esta sangre doblemente usada es devuelta al gemelo bomba a través de anastomosis venovenosas reduciendo el nivel de oxigeno, 
causando hipoxia crónica y restricción de crecimiento, demandando un alto gasto cardiaco.

Los contribuyentes que llevan a la muerte perinatal del gemelo bomba son la falla cardiaca congestiva y el parto prematuro ${ }^{(3)}$. El promedio de edad gestacional al parto de los gemelos acárdicos es de $31^{+1}$ semanas $\left(36^{+6}\right.$ semanas promedio en embarazos gemelares). El riesgo de complicaciones es más alta en el tercer trimestre cuando la tasa de crecimiento es mayor ${ }^{8}$.

\section{Clasificación}

Existen varias clasificaciones para los gemelos acárdicos. La más simple los divide en 2 tipos:

1. Pseudoacárdico: el gemelo tiene evidencia de una estructura cardiaca primitiva.

2. Holoacárdico: cuando dicha estructura no existe.

Otra clasificación basada en la morfología:

1. Acárdico acéfalo, donde el feto tiene bien desarrollado la pelvis $y$ extremidades inferiores, pero no la cabeza, órganos torácicos ni extremidades superiores.

2. Acárdico anceps, donde el feto tiene cuerpo bien desarrollado pero cabeza y cara parcialmente formadas. Es la forma mejor desarrollada.

3. Acárdico acormus, donde sólo la cabeza del feto se desarrolla. Poco frecuente.

4. Acárdico amorfo, donde el feto constituye una masa sin forma ni órganos reconocibles, pero con una estructura axial. Forma menos diferenciada.

Este sistema se utilizaba en el pasado, pero no guarda relación con el resultado perinatal ni con implicaciones terapéuticas, desarrollado en un tiempo donde no existía diagnóstico prenatal, por lo tanto se basaba en hallazgos postnatales. Ahora con el ultrasonido se intenta clasificar según el tamaño del feto y su impacto en el gemelo bomba ${ }^{(2)}$.

\section{Diagnóstico prenatal}

Debido a la mortalidad del 55\% del gemelo bomba, es importante diagnosticarlo lo más pronto posible para poder tomar medidas terapéuticas para mejorar la sobrevida. El diagnóstico prenatal debe sospecharse al detectar un feto severamente malformado en un embarazo gemelar monocorial. Entre las características ultrasonográficas se encuentra: discordancia entre el tamaño de los fetos, ausencia de latidos cardiacos, escasa definición de la cabeza, tronco y extremidades superiores, deformidad de extremidades inferiores, edema subcutáneo marcado difuso y aéreas quísticas anormales en la mitad superior de gemelo afectado.

La presencia de motilidad cardiaca no excluye el diagnóstico debido a que puede ser un corazón rudimentario o pulsación trasmitida por el gemelo bomba. La demostración de circulación con dirección paradojal con flujo arterial hacia el gemelo acárdico y flujo aórtico con dirección hacia cefálico en vez de caudal, establece el diagnóstico. Este flujo retrógrado se puede observar por ultrasonido Doppler ${ }^{(2,3,5)}$.

El principal diagnóstico diferencial es la muerte intrauterina de un gemelo monocorial severamente malformado. Sin embargo el continuo crecimiento en exámenes sucesivos debe sugerir la anomalía. Deben descartarse también teratoma placentario o del cordón umbilical. El gemelo acárdico muestra alguna organización corporal, mientras que el teratoma los tejidos están completamente desorganizados. En el periodo postnatal, la distinción se hace porque el acárdico está cubierto por piel normal $^{(2)}$.

\section{Características pronósticas}

Los factores predictores del resultado perinatal del gemelo bomba son determinados principalmente por la estructura del gemelo acárdico. El tamaño relativo del gemelo acárdico comparado con su co-gemelo juega el rol más importante $^{(5)}$. Si la relación de peso es más del $70 \%$, la incidencia de parto prematuro es de un $90 \%$, polihidramnios en un $40 \%$ y falla cardiaca congestiva en un $30 \%$, con tasas de $75 \%, 30 \%$ y $10 \%$ respectivamente, cuando la relación es menos del $70 \%{ }^{(9)}$. Los mejores resultados se 
presentan cuando la relación es menor a un 50\%. Pero estas mediciones han sido postnatales, lo cual significa que no son tan útiles en el diagnóstico prenatal, debido a que el tamaño del feto puede fluctuar significativamente in utero.

Es difícil hacer una relación peso prenatal por la estructura amorfa del gemelo acárdico.

Se ha utilizado el ultrasonido Doppler para medir y analizar la hemodinamia de ambos fetos, para identificar factores pronósticos. Los índices de impedancia en la arteria uterina son los principales, donde niveles bajos representan una alta velocidad diastólica en el acárdico, lo que se correlaciona con un mal resultado perinatal, debido a que refleja un importante flujo hacia el interior del acárdico. También se ha visto que diferencias entre los índices de resistencia de ambos gemelos juegan un papel importante, ya que pequeñas diferencias indican una gran comunicación arterio-arterial y un gran volumen de sangre desde el gemelo bomba al gemelo acárdico, con el consiguiente riesgo de falla cardiaca $^{(2,5)}$.

Aunque faltan varios estudios para poder determinar con certeza la utilidad pronóstica de estos métodos, se sabe que el tamaño del feto y el status cardiovacular del gemelo bomba son los factores más importantes para predecir el resultado perinatal y orientar el manejo. Otro factor que hay que tomar en consideración es la velocidad de crecimiento del acárdico, ya que un crecimiento acelerado indica un incremento en el gasto cardiaco del gemelo bomba. Al contrario, un disminución del tamaño indica una reducción del flujo, lo que puede llevar a una resolución espontánea del problema.

Se puede utilizar métodos para buscar signos del status cardiovascular del gemelo bomba. Mediante ultrasonido se pueden buscar signos físicos de deterioro cardiovascular como polihidramnios, cardiomegalia y derrame pericardico. Con el Doppler color para buscar regurgitación tricuspídea, flujo reverso en el ductus venoso, pulsatilidad en la vena umbilical y velocidad de flujo elevado en arteria cerebral media secundario a anemia fetal ${ }^{(2)}$.

Basándose en estos datos se ha creado una clasificación, considerando la severidad del caso y la necesidad de terapia prenatal. Se clasifican en dos tipos: tipo I si la relación de peso es menor a $50 \%$ y tipo II si la relación es mayor a $50 \%$. Estos se subdividen en "a" y "b" según la ausencia o presencia de signos de insuficiencia cardiaca.

\section{Tratamiento}

El tratamiento tiene como meta alcanzar la sobrevida del feto bomba e idealmente un parto de término. Se puede tener un manejo expectante solo si no encontramos factores de mal pronóstico, de los cuales el tamaño del gemelo acárdico es uno de los más importantes ${ }^{(2,5)}$. En el caso que el US demuestre factores de riesgo, el tratamiento prenatal está justificado. Se ha descrito numerosa.s técnicas para obliterar el flujo sanguíneo ${ }^{(10)}$, pero al ser este un evento infrecuente, ninguna técnica ha demostrado ser mejor.

Se ha descrito realizar histerotomía, con parto selectivo del gemelo acárdico. Aunque ha demostrado tener buena sobrevida para el gemelo bomba, este procedimiento conlleva un alto riesgo materno. Debido a estos riesgos no se recomienda sobre otras técnicas menos invasivas. En este momento existen técnicas mínimamente invasivas que aportan mejores resultados para el gemelo bomba con los menores riesgos maternos. Estos tratamientos se pueden dividir en intervenciones guiadas por ultrasonido $\mathrm{y}$ en guiados por fetoscopía.

El tratamiento se dirige a la oclusión del cordón umbilical o a la oclusión de los vasos intrafetales. En la oclusión del cordón se ha visto mejores resultados antes de la semana 24 (16-24 semanas $)^{(2,3)}$. Se ha asociado una tasa de fallo del $10 \%$ y entre sus riesgos se encuentran la ruptura de membranas, infección intra-amniótica y sangrado del gemelo normal, además de la obliteración del cordón del gemelo normal. Las técnicas intrafetales se dirigen hacia la aorta abdominal o a los vasos pélvicos del gemelo acárdico, los cuales son fácilmente identificables. Comparando estas dos técnicas, se ha visto mejor resultado y mayor tasa de éxito con la oclusión intrafetal, por lo que debería ser el tratamiento de elección $^{(2)}$. 
En cuanto a la oclusión del cordón umbilical, la técnica "gold standard" es la oclusión por coagulación bipolar, por su corta duración y sus disminuidos riesgos ${ }^{(2)}$. El principal riesgo lo determina el grosor del cordón umbilical que puede llevar a sangrado del cordón. En varios estudios donde se analizaron los resultados de dichas cirugías, se determinó entre sus riesgos: fallecimiento fetal intrauterino, ruptura prematura de membranas, parto prematuro y morbimortalidad neonatal. La ruptura prematura de membranas se define como el evento que se produce en las primeras 4 semanas post-cirugía, con una incidencia de $22-23 \%^{(2)}$ y el fallecimiento fetal en las primeras 2 semanas post-cirugía, con una incidencia de $15 \%{ }^{(2)}$. La muerte neonatal se presentó en un $4-8 \%^{(2)}$ de los casos, principalmente por la prematuridad. Estos resultados van en conjunto con la edad gestacional en la que se realiza el feticidio. Al final, la tasa global de sobrevida del bebé es de $79-83 \%, 4$ a 6 veces mayor que antes (de $21 \%$ a $79 \%)^{(2)}$.

\section{CONCLUSIONES}

La secuencia de perfusión arterial reversa (TRAP) es una malformación congénita exclusiva de gemelos monocoriales. Es poco frecuente pero debe ser diagnosticada de forma temprana para poder brindar el tratamiento óptimo y así mejorar el pronóstico del gemelo bomba.

Con el paso del tiempo se ha desctrito nuevas técnicas terapéuticas, que tienen mejor pronóstico perinatal para la madre y el feto, sin embargo debido a lo poco frecuente de la entidad, hacen falta más estudios para definir cuál es el tratamiento óptimo.

Actualmente, la oclusión del cordón umbilical o la oclusión de los vasos intrafetales es la que ha demostrado mejores resultados.

\section{BIBLIOGRAFÍA}

1. Cunningham F. Wiliams Obstetrics, $23^{\text {rd }}$ Ed. 2005, McGraw-Hill.

2. Sepúlveda W Wong A Pons A Gutiérrez G Corral E. Secuencia de perfusión arterial reversa (gemelo acárdico): Evaluación prenatal $y$ tratamiento. Revista Chilena Ultrasonografía. 2005;8:118-130.

3. Lewi L Valencia C González E Deprest J Nicolaides K. The outcome of twin reversed arterial perfusion sequence diagnosed in the first trimester. American Journal Obstetrics and Gynecology. 2010;203(3):213e1-e4.

4. Van Allen M Smith DW Shephard T. Twin reversed arterial perfusion (TRAP) sequence: a study of 14 twin pregnancies with acardius. Seminars in Perinatology 1983;7:285-293.

5. Bornstein E Monteagudo A Dong $\mathrm{R}$ Schwartz N Timor-Tritsch IE. Detection of Twin Reversed Arterial Perfusion Sequence at the Time of First-Trimester Screening. Journal of Ultrasound in Medicine 2008;27:1105-1109.

6. Severn C Holyoke E. Human acardiac anomalies. American Journal Obstetrics and Gynecology 1973;116:358-365.

7. Coulam C Wright G. First trimester diagnosis of acardiac twins. Early Pregnancy 2000;4:261-270.

8. Healey M. Acardia: predictive risk factors for the co-twin's survival. Teratology 1994;50:205-213.

9. Moore T Gale S Benirschke K. Perinatal outcome of forty-nine pregnancies complicated by acardiac twinning. American Journal Obstetrics and Gynecology 1990;163:907-912.

10. Robyr R Yamamoto M Ville Y. Efficacy of radiofrequency ablation for twin-reversed arterial perfusion sequence. International Journal of Obstetrics and Gynecology. 2005;112:1344-1348.

\section{CONFLICTO DE INTERÉS}

Los autores declaran que no existe ningún conflicto de interés en el presente reporte. 